\title{
Controversias sobre el ejercicio profesional de la psicología en Uruguay
}

\author{
Jorge Chavez, (iD) I, Paula Martínez Sena (iD) II \\ Universidad de la República, Montevideo, Uruguay
}

\begin{abstract}
Resumen
La psicología como profesión tiene una historia breve en comparación a otras disciplinas del ámbito de la salud y especialmente de la salud mental. La legitimación de su práctica profesional en el Uruguay, ha sido un proceso controvertido y disputado con otras disciplinas, particularmente la psiquiatría. En este artículo nos proponemos estudiar las acciones que diferentes actores desarrollaron para delimitar un campo de actividad profesional entre 1958 y 1984. Se optó por una metodología cualitativa apostando al análisis e interpretación de fuentes bibliográficas relevantes desde los aportes de la historia crítica de la psicología y de la sociología de las profesiones. Las fuentes analizadas se componen de informes técnicos jurídicos, boletines y actas de agrupaciones de psicología y psiquiatría, revistas y documentos universitarios como expedientes y planes de estudio. Se concluye que más allá de las disputas por los modos de circulación, apropiación y aplicación del conocimiento sobre contenidos específicos como la psicoterapia, la lucha se entabla por campos de ejercicio mucho más amplios que emergen a la vez que los diferentes actores movilizan sus intereses. Palabras clave: profesión; ejercicio profesional; reglamentación; controversias.
\end{abstract}

\section{Controversies about the professional practice of psychology in Uruguay}

\begin{abstract}
Psychology as a profession has a short history compared to other disciplines in the field of health and especially mental health. The legitimation of it's professional practice in Uruguay has been controversial and disputed with other disciplines, especially psychiatry. In this article we propose to study the actions taken to delimit the field of professional activity during 1958 to 1984 . We chose a qualitative methodology, using the analysis and interpretation of relevant bibliographic sources from the contributions of the critical history of psychology and the sociology of the professions. The analyzed sources are legal technical reports, bulletins and minutes of psychology and psychiatry groups, magazines and university documents such as files and study plans. It is concluded that beyond the disputes over the ways of circulation, appropriation and application of knowledge on specific contents such as psychotherapy, the struggle is fought over much broader fields that emerge at the same time that the different actors mobilize their interests.
\end{abstract}

Keywords: profession; professional practice; regulation; controversies.

\section{Controvérsias sobre a prática profissional da psicologia no Uruguai}

A psicologia como profissão tem uma história curta em comparação com outras disciplinas da área da saúde e principalmente da saúde mental. A legitimação de seu exercício profissional no Uruguai tem sido um processo polemico e disputado com outras disciplinas, em particular a psiquiatria. Neste artigo propomos estudar as ações que diferentes atores desenvolveram para delimitar um campo de atividade profissional entre 1958 e 1984. Optou-se por uma metodologia qualitativa, apostando na análise e interpretação de fontes bibliográficas relevantes a partir das contribuições da história crítica da psicologia e da sociologia das profissões. As fontes analisadas são compostas por relatórios técnicos jurídicos, boletins e atas de grupos de psicologia e psiquiatria, revistas e documentos universitários, como arquivos e planos de estudos. Conclui-se que para além das disputas pelos modos de circulação, apropriação e aplicação do conhecimento em conteúdos específicos como a psicoterapia, a luta se trava em campos de exercício muito mais amplos que surgem à medida que os diferentes atores mobilizam os seus interesses.

Palavras-chave: profissão; prática profissional; regulação; controvérsias.

\section{Introducción}

El ejercicio profesional de la psicología en Uruguay se legisló en 1999, luego de un controvertido proceso iniciado en la década de 1960, en el que participaron actores con visiones e intereses contrapuestos. Los debates en torno a qué acciones son legítimas en el ejercicio de la psicología y cuáles son los límites de su campo de acción, se actualizan cada vez que se intenta regular un espacio fronterizo entre las disciplinas "psi”, como sucedió con en el reciente proceso de elaboración y promulgación de la Ley de Salud Mental en agosto del 2017.

\footnotetext{
^Dirección para correspondencia: Facultad de Psicología, Universidad de la República. Dr Tristán Narvaja 1674, 11200. Montevideo, Uruguay. E-mails: jorgechavezpsic@gmail.com,pmartinezsena2@gmail.com

Los datos completos de los autores se pueden encontrar al final del artículo.
}

En este artículo nos proponemos describir y analizar las acciones que diferentes actores desarrollaron buscando delimitar un campo de actividad profesional para la psicología, apoyándonos en los desarrollos teóricos de la sociología de las profesiones y de la historia crítica de la psicología.

La sociología de las profesiones estudia el modo en que diferentes disciplinas negocian, se enfrentan y definen sus identidades, excluyendo a otras "tribus académicas" (BECHER, 2001), o el modo en que construyen al profesional a la vez que profesionalizan la disciplina, especializándose en un conjunto de tareas fundamentadas teóricamente que permiten organizar la división del trabajo, el control del mercado y la enseñanza de dicha profesión (FREIDSON, 1996). Se entiende que las profesiones pueden poseer un territorio delimitado, que puede ser invadido, colonizado o resignado (BECHER, 2001). Abbott (1988) plantea que otras profesiones pueden re- 
clamar o pretender esos territorios, pudiendo ocupar las jurisdicciones cuando se encuentran vacantes o luchando por ellas. Define jurisdicción como la conexión que vincula a una profesión con el trabajo que realiza y puede ser conquistada por una profesión en dos tipos de arenas; una formal: el espacio público y legal; y otra informal: el lugar de trabajo. Es en el ejercicio concreto donde la profesión adquiere rasgos de singularidad: define y controla sus tareas, construye la cultura de trabajo donde aplica los conocimientos, la producción y enseñanza de los mismos. Estos rasgos singulares refieren a competencias específicas para el trabajo en un campo determinado, y habilitan a reivindicar potestad sobre el mismo. La autodefinición de un grupo como legítimos agentes profesionales y de su campo de intervención, resultan de acciones complementarias motivadas por intereses que inciden en la construcción de su identidad social (ABBOTT, 1988).

Desde la historia crítica de la psicología tomamos los aportes de Kurt Danziger y Nikolas Rose. Danziger (1984) propone concebir como sujetos colectivos a los grupos que movilizan intereses, estando quienes intentan legitimar una disciplina y quienes ocupan posiciones de jerarquía y distribuyen recursos materiales vinculados a la transmisión, aplicación y producción del conocimiento. El estudio de las acciones de los sujetos colectivos que disputan la preeminencia sobre el campo 'psi', legitimando habilidades y delimitando un campo de ejercicio, nos permite elucidar lo que Rose (1998) llamó: profesionalización de la psicología.

\section{Metodología}

Este artículo es producto de la investigación en curso titulada: Historia crítica de la psicología en el Uruguay: 1960-1984. Se optó por una metodología cualitativa, apostando al análisis e interpretación de fuentes bibliográficas relevantes desde los aportes de la historia crítica de la psicología y la sociología de las profesiones. Se agruparon las fuentes correspondientes a tres períodos: de 1968 a 1975 , de 1975 a 1979 y de 1979 a 1984, considerando los momentos en los que diversas propuestas de reglamentación entraron en disputa, y enfocando el análisis en las acciones y argumentos que los colectivos realizaron para justificar o consolidar sus posiciones. El corpus de fuentes analizado se compone de informes técnicos jurídicos, boletines y actas de agrupaciones de psicología y psiquiatría, revistas y documentos universitarios como expedientes y planes de estudio, entre 1958 y 1984. El objetivo es describir y analizar las acciones que ciertos sujetos colectivos realizaron en torno a las propuestas de reglamentación del ejercicio profesional de la psicología, previo y durante la dictadura cívico-militar en Uruguay (1958-1984) y avanzar sobre la comprensión de los modos de relacionamiento entre disciplinas en el complejo campo de la salud mental.

\section{Bases para el ejercicio profesional de la psicología}

La creación de los cursos universitarios de formación en psicología a mediados de la segunda mitad del siglo $\mathrm{XX}$, marcaron un punto de inflexión en la organización y regulación del ámbito de las prácticas “psi”, habitado por psiquiatras, psicoanalistas en formación y actores interesados en asumir la identidad profesional de psicólogo.

En 1950, comenzó el Curso de Psicología Aplicada a la Infancia formando Técnicos en Psicología Infantil en la Facultad de Medicina, a los que se instruía en tareas asistenciales (MARCOS, 1949), construyendo un quehacer profesional caracterizado por la colaboración subordinada al ejercicio médico. El otro espacio universitario fue la Licenciatura en Psicología en la Facultad de Humanidades y Ciencias (en adelante FHC), que desde el inicio fue un territorio a disputar. En el año 1951, el decano de FHC Justino Jiménez de Aréchaga, le solicitó al Dr. Horacio Rimoldi ${ }^{1}$ crear la licenciatura de psicología. El proyecto de Rimoldi, con un fuerte direccionamiento sobre la psicología estadística, fue resistido por actores locales del ámbito médico-universitario, provocando que desistiera de ejecutarlo en 1953 (CHAVEZ, 2020; OIBERMAN, 2001).

La licenciatura en psicología se creó en 1956 a cargo del médico psiquiatra Mario Berta. La formación de grado tuvo perfil experimental, incluyendo perspectivas clínicas que acompañadas por el influjo de la formación psicoanalítica que se iniciaba en nuestro país, alteraron el objetivo formativo de la licenciatura. La FHC tenía un enfoque académico centrado en la investigación e incluyó un perfil profesionalista para la psicología, generando la posibilidad de que la clínica psicoterapéutica integrara las prácticas en el futuro ejercicio profesional (CHAVEZ, 2020).

La formación universitaria y la incorporación de la clínica psicoterapéutica en la práctica profesional mostraban el camino hacia la autonomía académica y profesional, una meta que identificó y nucleó a quienes en 1953 crearon la novel Sociedad de Psicología del Uruguay $^{2}$ (en adelante SPU). La psicología hasta ese momento no resultaba una amenaza para otras profesiones, ya que su jurisdicción se concentraba en actividades de asistencia a la medicina o investigación en el marco de la psicología experimental. Sin embargo, el estudio y la práctica del psicoanálisis junto al proceso de su institucionalización en la órbita internacional, tuvieron una relevancia singular. El psicoanálisis fue recepcionado por médicos y legos, mixtura que aportó un carácter híbrido a su institucionalización, que al avanzar sobre el espacio profesional generó posturas de oposición en cierto sector de la psiquiatría local (LIJTENSTEIN; VIÑAR, 1992).

En la sesión 27 de mayo de 1958, la Sociedad de Psiquiatría del Uruguay abordó el tema: "El intrusismo en psiquiatría". El Dr. Reyes Terra disertó sobre el problema de la psicoterapia ejercida por los psicólogos clínicos, psicoterapeutas y psicoanalistas no médicos, argumen-

\footnotetext{
${ }^{1}$ Horacio Rimoldi (1913-2006) fue una figura destacada de la psicología iberoamericana que tuvo su primer contacto con Uruguay al participar de la investigación sobre el 'normotipo del niño y del adolescente uruguayo', dirigida por el Doctor Emilio Mira y López entre abril de 1944 y setiembre de 1945.

${ }^{2}$ La SPU fue creada el 7 de diciembre de 1953 a iniciativa de un grupo de psicólogos, psiquiatras y estudiosos de las ciencias 'psi'. En 1957 se creó un grupo de trabajo con el fín "de estudiar los problemas de la creación de la profesión de psicólogo" (SPU, 1973, p. 2) concretando el primer proyecto en 1967 que fue elevado a la UdelaR.
} 
tando la incapacidad e inhabilitación de los mismos para ejercer el arte de curar, esgrimiendo motivos legales, gremiales y técnicos. Sostuvo que en caso que realizaran esta tarea, serían considerados infractores legales (REYES TERRA, 1958a). El 12 de junio de 1958, miembros de la Sociedad de Psiquiatría del Uruguay en una nueva acción para limitar la actividad de lo que llamaban intrusos, fueron recibidos en audiencia por el Ministro de Salud Pública el Dr. Vicente Basagoity. Reyes Terra expresó que la formación en FHC se orientaba hacia la investigación, por lo que sus egresados no podían ejercer la psicoterapia pero sí operar como técnicos colaboradores del médico. Respecto a los analistas y por la singularidad de su técnica en cuanto al manejo de la transferencia que necesita de autonomía absoluta, sostuvo que esa autonomía es inexistente debido a que dependen del médico (REYES TERRA, 1958b).

La defensa de la jurisdicción médica alcanzó al Instituto de Psicología de la FHC en la figura de su Director, el psiquiatra Dr. Mario Berta, a quien la Comisión Directiva de la Sociedad de Psiquiatría del Uruguay solicitó un informe sobre los alcances del ejercicio profesional de los psicólogos. En respuesta, Berta evaluó la relación psicología-medicina, estableciendo sus características, relaciones jerárquicas y campo de trabajo. Aún mencionando áreas específicas de competencia del psicólogo y la validez de su formación universitaria, reduce su figura y quehacer a la de un técnico que aplica tests y orienta. El tratamiento a los enfermos queda fuera de las consideraciones legales de la época, ya que esta tarea estaba reservada a los médicos y propuso como solución al problema del ejercicio de la psicoterapia, la creación de una Escuela Universitaria de Psicoterapia a la que podrían acceder Psicólogos y Médicos con título universitario (BERTA, 1958). Propuesta que jamás se concretó.

En 1960, la SPU organizó las primeras Jornadas Uruguayas de Psicología, donde tuvo lugar una Mesa Redonda sobre la Profesión del Psicólogo. En dicha ocasión, Juan Carlos Carrasco ${ }^{3}$ cuestionó duramente el monopolio de la práctica terapéutica, interpelando la idea de exclusividad de la oferta en el mercado de los servicios en salud mental. En 1966, Berta renunció a la dirección de la licenciatura debido a presiones de índole política y académica, lo que debilitó la preeminencia médica en la formación en psicología de la FHC. El cargo fue ocupado por el médico y psicoanalista Jorge Galeano Muñoz, durante poco tiempo, ya que en 1968 asumió Juan Carlos Carrasco. Estos acontecimientos expresan un proceso de transformaciones tendientes a la construcción de una psicología autónoma, a los que se sumó el intento por reglamentar la profesión y la adecuación de los contenidos en la formación de grado.

\footnotetext{
Juan Carlos Carrasco (1923-2010) fue pionero en la consolidación de la psicología universitaria. Director del Instituto de Psicología de la FHC hasta que debió exiliarse al inicio de la dictadura cívico militar en 1973. A su regreso promovió junto a otros actores, la unificación de los espacios de formación universitaria en psicología, fue director del novel Instituto de Psicología de la Universidad de la República, espacio unificado de formación, previo a la creación de la facultad de psicología en 1994. Nombrado Profesor Emérito de la Facultad de Psicología.
}

En 1967 la SPU consultó al Ing. Oscar Maggiolo, Rector de la Universidad de la República (en adelante UdelaR) sobre la posibilidad de incluir la creación de la carrera de psicología dentro de la Ley Orgánica. La respuesta afirmativa llevó a presentar el primer Anteproyecto de Reglamentación de la Profesión del Psicólogo. Este intento inaugural de delimitar la jurisdicción de la psicología no prosperó, y si bien reclamó la libertad para realizar psicoterapia, mantuvo la condición de aprobación médica previa al inicio del proceso terapéutico (SOCIEDAD DE PSICOLOGÍA DEL URUGUAY [SPU], 1983).

En 1968, el nuevo plan de estudios de la licenciatura incluyó contenidos de psicoanálisis y psicología social dejando atrás enfoques más estadísticos y experimentales, a la vez que se abrieron nuevos ámbitos de ejercicio como el trabajo con la comunidad (CHAVEZ; IRRAZABAL, 2018). Las prácticas en los barrios fueron el vehículo para conectar estudiantes y docentes con la compleja realidad nacional del momento. En el cierre de las Primeras Jornadas Nacionales de Psicología Infantil, las palabras de Juan Carlos Carrasco (1967) marcaban el rumbo que debía tomar la psicología universitaria: "La psicología además tiene firmado otro compromiso. Este compromiso es con la comunidad. La psicología sin proyección comunitaria corre el riesgo de transformarse en un juguete de lujo en manos de privilegiados" (CARRASCO, 1967, p. 181).

\section{Primeras propuestas de reglamentación y controversias sobre las acciones del psicólogo (1968- 1975)}

El final de la década de 1960 presentó un ambiente social y político complejo: una grave situación económica fue intensificando el descontento social, dando lugar a fuertes conflictos laborales, huelgas y evidenciando la incapacidad del sistema político para encontrar soluciones a los problemas del país. En 1968 se aplicaron por primera vez las Medidas Prontas de Seguridad con el argumento de defender el orden público y la tranquilidad interior. La lucha contra la guerrilla urbana y el estado de excepción se volvieron una constante hasta que en 1972 la Asamblea General aprobó la suspensión de las garantías individuales y declaró el estado de guerra interno (RICO et al., 1996).

En ese contexto, el 28 de setiembre de 1970 el Consejo Directivo Central de la UdelaR aprobó el nuevo plan de estudios para la Licenciatura de Psicología de la FHC, que comenzó a funcionar en 1971. La propuesta incluyó estudios de grado, especializaciones, maestría y doctorado, evidenciando el fortalecimiento de la psicología como disciplina independiente y tendiendo a legitimar su accionar profesional como ya lo hacía con la formación.

Ante ese avance, las respuestas desde la psiquiatría no se hicieron esperar. Ese mismo año, el Dr. Tobler, Profesor Adjunto de Psiquiatría y Director del Departamento de Psiquiatría e Higiene Mental del Ministerio de Salud Pública (en adelante MSP), elevó a este organismo una propuesta incluyendo directrices para establecer normas tendientes a evitar el ejercicio ilegal de la psicología, con 
el título: "Nuevas normas para el ejercicio de psicólogo", incluyendo también las Normas para el ejercicio de Técnico en Psicología Infantil. En una carta dirigida al Prof. Dr. Pablo Purriel, Ministro de Salud Pública en ese entonces, el Dr. Tobler señaló la carencia de reglamentación para las actividades específicas de psicólogos y psicotécnicos, lo que favorecía una serie de 'anomalías' como "el ejercicio de la profesión sin la debida preparación; extralimitación de funciones; invasión por parte de extranjeros, que no pudiendo ejercer en el propio país, recurren al nuestro por su liberalidad en esta materia" (FACULTAD DE MEDICINA, 1975, p. 2). Con respecto a las funciones del psicólogo, la normativa habilitaba el uso de métodos y técnicas psicológicas, diagnóstico, orientación, selección profesional y solución de problemas de conducta, puntualizando que la terapia debía ejercerse bajo control del médico especialista. Planteaba que la formación de psicólogos debía realizarse en la FHC y para los Psicotécnicos Infantiles, en la Escuela Colaboradores del Médico ${ }^{4}$ de la Facultad de Medicina, debiendo actuar bajo el contralor y por indicación del médico especialista en Psiquiatría.

En julio de 1972, la Dra. Ofelia Bachini, Coordinadora del Servicio Psiquiátrico del MSP, introdujo modificaciones al proyecto y en setiembre, el Asesor Letrado del MSP Dr. Santos Barriere consideró necesario conocer la opinión de la Licenciatura en Psicología de la FHC y de la Escuela de Colaboradores del Médico sobre la propuesta del Dr. Tobler y el MSP. Para esto remitió el expediente a la UdelaR. El Consejo de la FHC en la sesión del 19 de octubre de 1972, dispuso trasladar la propuesta de reglamentación al Instituto de Psicología y a la Asociación de Egresados de Psicología para su consideración (FACULTAD DE MEDICINA, 1975).

En noviembre, una Comisión Asesora del Instituto de Psicología ${ }^{5}$ analizó la propuesta considerándola satisfactoria si se incluían una serie de modificaciones: quienes debían homologar los títulos extranjeros para habilitar al ejercicio serían los organismos especializados en psicología de la FHC, el MSP otorgaría certificados habilitantes para el ejercicio a quienes hayan acreditado actividad profesional ininterrumpida durante cinco años en policlínicas psiquiátricas $\mathrm{u}$ hospitalarias y por último, para el ejercicio de la psicoterapia, se propuso como requisito previo la realización de un examen médico (FACULTAD DE MEDICINA, 1975).

A finales de 1972 e inicios de 1973, los colectivos de psicología estuvieron alertas y movilizados. La SPU convocó para el 18 de enero a las 19:30 hs. a una Asamblea General Extraordinaria que levantaba el cuarto intermedio de la Asamblea realizada el 28 de diciembre de 1972, con un único punto en el orden del día: Anteproyecto de ley para el ejercicio de la profesión de psicólogo, una nueva propuesta elaborada por la SPU que salía al cru-

${ }^{4}$ La Escuela de Colaboradores del Médico fue el espacio en donde se desarrolló la formación de los Técnicos en Psicología Infantil, continuaba la experiencia del Curso de Psicología Aplicada a la Infancia de la Clínica Médico Psicológica del Hospital de niños Dr. Pedro Visca.

${ }^{5}$ A la Comisión Asesora asistieron los licenciados Carlos Sopena, Leopoldo Muller, Fernando Arias, Eliseo González, Carmen Hernández, Rina Valeta, los estudiantes Eleonora Perez, Hector Secco y la profesora Nelda Cajigas de Segredo. ce del proyecto del MSP (SPU, 1973). Previo a la primer asamblea, la SPU consultó al Dr. Justino Jiménez de Aréchaga $^{6}$ y al Dr. Horacio Cassinelli Muñoz ${ }^{7}$ sobre las características jurídicas de la propuesta del Dr. Tobler y el MSP. EL 27 de diciembre en una carta dirigida a Elida Tuana $^{8}$, Cassinelli Muñoz informó que el proyecto del MSP se aplicaría para el caso en que la psicología fuese entendida como una actividad 'auxiliar' a la medicina, mientras que consideró ilegales "las disposiciones que pretenden reservar exclusivamente a los titulados registrados aquellas funciones del psicólogo que no tienen carácter médico ni paramédico" (CASSINELLI, 1972, p. 1). El informe de Jiménez de Aréchaga del 29 de diciembre de 1972, expresó que: "El proyecto suscripto por el Dr. Tobler es de una extrema vaguedad" (JIMENEZ DE ARECHAGA, 1972, p. 1) y que para restringir la libertad de trabajo, la misma debe ser impuesta a través de una ley y no de un decreto. Agrega que al tratarse de la creación de una nueva profesión liberal la normativa debe regular la actividad de los psicólogos dentro y fuera del MSP y que este ministerio no tendría que estar a cargo de dicha regulación, sino que actuaría solamente en los casos de ejercicio de psicoterapeutas o vinculados a la salud de los clientes (JIMENEZ DE ARECHAGA, 1972).

En la Asamblea Extraordinaria del 18 de enero de 1973, la SPU advirtió que el proyecto del MSP desconoce la actividad profesional de los psicólogos fuera del ámbito psicoterapéutico, como las tareas realizadas en el ámbito comunitario, educacional o laboral. En base a lo informado por los juristas, calificó como inaceptable reglamentar una profesión que no existe y reclamó que se proceda bajo las mismas normas con las que se actuó sobre otras profesiones, amparando a quienes ya ejercían las tareas del oficio. La SPU propuso:

$1^{\circ}$ ) se realicen los actos necesarios para que por ley se cree la profesión de psicólogo en la República y se establezca el monopolio de la misma;

$2^{\circ}$ ) un proyecto de ley que crea y reglamenta la profesión de psicólogo cuyo texto adjunta (SPU, 1973, p. 2).

La propuesta impulsada por algunos psiquiatras proveniente del MSP, intentó reglamentar el ejercicio de la profesión sin reconocerla como existente, lo que lleva a considerar que la motivación de tal acción apuntaba a defender un espacio concreto de la jurisdicción médica. El interés de establecer que la psicología era una actividad auxiliar de la función médica, contribuía a no reconocer su independencia y a deslegitimar actividades que expresaran alguna cualidad de disciplina autónoma.

La SPU propuso un Anteproyecto de ley para el ejercicio de la profesión de Psicólogo a través del que se creaba y reconocía oficialmente la profesión, estableciendo a la UdelaR como espacio exclusivo de formación

${ }^{6}$ Justino Jiménez de Aréchaga (1910-1980) fue un importante jurista que ocupó destacadas posiciones en diferentes espacios institucionales nacionales e internacionales. Fue Profesor Emérito, Facultad de Derecho y Ciencias Sociales y Decano de la Facultad de Humanidades y Ciencias (1951).

${ }^{7}$ Horacio Cassinelli Muñoz (1931-2014) fue Profesor emérito de Derecho Constitucional y Derecho Público, sus obras son de referencia a nivel jurídico y universitario en Uruguay.

${ }^{8}$ Elida Tuana (1913-2005) en 1972 ocupaba la presidencia de la SPU. 
profesional. El ejercicio se habilitaba para quienes contaban con título de la Universidad, revalidado en el caso de los extranjeros y reconocido en el caso de quienes venían ejerciendo previamente. En cuanto a las tareas que buscaron monopolizar, se encuentran el uso de "métodos y técnicas psicológicas con fines de diagnóstico, orientación y asistencia en los campos comunitario, institucional, educacional, laboral, familiar e individual" (SPU, 1973, p. 2). Para la psicoterapia, se entendía que necesita de un enfoque interdisciplinar, y "podrá ser realizada por los psicólogos en la medida en que su labor surja del resultado de la interconsulta médico-psicológica" (SPU, 1973, p. 2). La SPU planteó la creación de una comisión asesora para la UdelaR, compuesta por miembros del Consejo Central Universitario, el Director del Instituto de Psicología de FHC, el Director del curso de Técnicos en Psicología Infantil de la Escuela de Colaboradores del Médico, el Catedrático de Psicología Médica de la Facultad de Medicina, y delegados del MSP, Ministerio de Cultura, SPU y la Asamblea de Interesados.

Ante esta nueva realidad, el 16 de febrero de 1973 , desde la Comisión Asesora del Instituto de Psicología, la Prof. Nelda Gajigas ${ }^{9}$ solicitó al decanato de la FHC la devolución del expediente al Instituto de Psicología. La presentación del proyecto de la SPU y los informes jurídicos recibidos, hicieron que el primer informe del Instituto de Psicología (7/11/72) perdiera validez. El nuevo informe (12/3/1973) elaborado por el Instituto fue más exhaustivo en su análisis y mucho más crítico que en su primera versión. Al informe se le adjuntó el anteproyecto de reglamentación elaborado por los egresados de la licenciatura nucleados en la Asociación de Psicólogos Universitarios del Uruguay (en adelante APUU), las modificaciones a este proyecto realizadas por el Instituto de Psicología, el proyecto elaborado por la SPU y los informes de los Dres. Justino Jiménez de Aréchaga y Horacio Cassinelli Muñoz. Un elemento distintivo en el proyecto de la APUU fue la referencia a la psicoterapia individual y grupal como modalidades de intervención clínica, manteniendo el requisito del previo examen médico. El informe elaborado por el Instituto de Psicología fue elevado el 22 de marzo al Consejo de FHC y el 16 de agosto el Decano Mario Otero lo derivó a la División Jurídica.

Entre esas fechas, el proceso de crisis del sistema político y del sistema democrático que venía desde 1967 precipita el golpe de Estado acaecido el 27 de junio de 1973. Se trató de un 'golpe institucional', el propio presidente del momento Juan M. Bordaberry devino "dictador de facto" (RICO, 2006, p. 16). Esto dio paso a una dictadura cívico-militar que duró hasta 1984. Rico (2006) destaca características singulares de la dictadura uruguaya. La primera, es que de inmediato desató una respuesta social y especialmente sindical de rechazo a través de una huelga general. Una segunda característica es que el golpe pasó por varias etapas en las que la represión y violencia fueron creciendo. Un ejemplo de esto es que en

\footnotetext{
${ }^{9}$ La Prof. Nelda Gajigas firmó los informes como Encargada de la Dirección del Instituto de Psicología.
}

setiembre de ese año se celebraron las elecciones universitarias y el 28 de octubre de 1973 la UdelaR fue intervenida, destituyendo autoridades y cientos de profesores.

Entre junio y octubre de 1973, algunas actividades universitarias continuaron, también el proceso de reglamentación de la profesión de psicólogo. En agosto de 1973 el Dr. Semino, Asesor Jurídico de la UdelaR, informó al Rector sobre el proyecto de reglamentación del MSP coincidiendo con las consideraciones de los juristas que asesoraron a la SPU. Sentenció que se trataba de una propuesta que buscaba "no sólo reglamentar y vigilar, sino también limitar una actividad hasta ahora libre" (FACULTAD DE HUMANIDADES Y CIENCIAS, 1973a). En cuanto al proyecto del Instituto de Psicología, dice que se entiende que ya existe la profesión de psicólogo y se trata de limitar su ejercicio por razones de interés general, postulando que el título habilitante es el obtenido en la UdelaR.

En el boletín de setiembre de la APUU (1973), la asociación instó a fortalecer el gremio, expectantes ante la posible reglamentación de la profesión. El boletín incluyó el "Proyecto sobre reglamentación de la profesión de psicólogo" redactado por la APUU, modificado por la Comisión Asesora del Instituto de Psicología de FHC y en estudio del Consejo Directivo Central de la UdelaR. El proyecto creaba la profesión de Psicólogo, pudiendo ejercerla los egresados de la UdelaR o aquellos formados en el extranjero y revalidados por los organismos especializados en Psicología de la FHC. Entre las funciones detalladas, se destacan el uso de métodos y técnicas psicológicas, la experimentación psicológica y psicofisiológica de la conducta, diagnósticos, psicoterapia grupal e individual con previo examen médico, asesoramiento y asistencia en psicoprofilaxis y psicohigiene, dirigir servicios y la enseñanza en psicología, así como brindar asesoramiento técnico a organismos, realizar pericias y opinar en materia de psicología (APPU, 1973).

El 9 de octubre de 1973, el Decano de FHC el Prof. Mario H. Otero, respondió a la solicitud realizada por el MSP sobre la propuesta de reglamentación de la profesión. La respuesta se centró en una definición: "hacer suyo el proyecto sobre Normas para el ejercicio de la profesión del Psicólogo presentado por el Instituto de Psicología de la Facultad en fecha 12/3/73" (FACULTAD DE HUMANIDADES Y CIENCIAS, 1973b, p. 1), incorporando los aportes del Dr. Semino e integrando los intereses de los colectivos de la psicología (SPU-APUU).

El 28 de octubre, el Poder Ejecutivo dispuso la intervención de la UdelaR con el decreto 921/973, la administración de la misma pasó al Ministerio de Educación y Cultura (en adelante MEC), se suspendió toda actividad docente y se dispuso el arresto de los miembros del Consejo Directivo Central. Con la FHC intervenida, las demás carreras volvieron al funcionamiento pero la Licenciatura de Psicología se cerró definitivamente, con docentes destituidos y estudiantes sin la posibilidad de concluir su formación en esa institución. La psicología universitaria sufría un fuerte revés que ponía en jaque su avance académico como profesional. 
Intervenciones, cierres, reglamentaciones y creaciones: construcción de un nuevo orden (1975-1979)

Hasta el momento de la intervención de la UdelaR, los psicólogos uruguayos se enfocaron sobre un doble objetivo; por un lado, resistir al establecimiento de un marco para el ejercicio de la profesión desde agentes vinculados a la psiquiatría y por otro, generar visibilidad sobre la profesionalización y la jurisdicción de la disciplina. Al intervenir la UdelaR, el gobierno de facto designó autoridades alineadas a sus intereses y para la psicología local, esto significó la instalación de un escenario poco auspicioso para los colectivos interesados en establecer la reglamentación del ejercicio profesional, abriendo además, un nuevo campo de conflictos referido a la formación en psicología y sus instituciones.

Durante la dictadura, el biólogo Miguel Klappenbach (1920-2000) fue designado como Decano Interventor de la $\mathrm{FHC}$, mientras que para dirigir una licenciatura que no volvió a abrir sus puertas, fue nombrado como Director del Instituto de Psicología el médico psiquiatra A. Sommer Smith. Ante esta designación, la Comisión Directiva de la SPU le solicitó una entrevista donde intercambiar ideas acerca de la reglamentación de la profesión y de los títulos en psicología. La SPU planteó su posición y el director interventor les entregó un nuevo proyecto: "Anteproyecto de ley de reconocimiento de la profesión de psicólogo". Este documento, como lo indica su título, reconocía la profesión de psicólogo como independiente y de nivel universitario, a ser ejercida por egresados del Instituto de Psicología de la FHC e institutos privados habilitados. Definía como áreas profesionales propias del psicólogo a la psicología clínica, educacional y de orientación, social y laboral, no así la psicoterapia. La SPU estudió el anteproyecto y objetó duramente que instituciones privadas otorgasen título para ejercer la profesión al mismo nivel que la UdelaR (SPU, 1974).

Las pujas en torno a la reglamentación continuaron entre dos sectores bien diferenciados: por un lado las instituciones intervenidas (UdelaR, MEC y MSP), en las que un sector de la psiquiatría local se fortaleció ocupando lugares de relevancia y por otro lado, los colectivos reunidos en torno a la SPU y la APUU. Los primeros buscaron convertir a la psicología en un conjunto de técnicas prefabricadas aplicadas bajo la supervisión médica; los segundos, buscaban crear un marco regulatorio donde el psicólogo ejerciera libremente su rol como profesional independiente. El escenario se volvió desfavorable para estos últimos, ya que la disputa rebasó el campo del ejercicio profesional para ocupar el ámbito de la formación. La educación en psicología resultó un campo central a disputar, controlar la transmisión de los conocimientos implicó el control sobre qué tipo de saberes y técnicas configurarían el quehacer de la profesión. Este control determinaría la construcción de los perfiles profesionales y sus características, delimitaría las fronteras de la jurisdicción para el ejercicio, y en definitiva demarcaría la legitimidad de lo que puede o no puede hacer el psicólogo.
La tecnicatura en Psicología Infantil de la Escuela de Colaboradores del Médico en la Facultad de Medicina, se mantuvo abierta a lo largo del período de facto, pero el cierre de los cursos en FHC preocupó a estudiantes por la falta de formación y la imposibilidad de concluir sus estudios. Esto llevó a que el Ministro de Educación Edmundo Narancio (1916-2001) dispusiera la creación de una comisión para estudiar si la Licenciatura en Psicología de FHC: "tiene el nivel técnico suficiente como para habilitar al ejercicio de esa profesión y además, la posibilidad de instalar una Escuela Universitaria de Psicología" (UNIVERSIDAD DE LA REPÚBLICA [UdelaR], 1975, no paginado). En agosto de 1974, el Prof. Gonzalo Fernández Decano Interventor de la Facultad de Medicina, creó una comisión ${ }^{10}$ integrada en su mayoría por médicos, para estudiar el programa de la Licenciatura de Psicología y las posibles modificaciones a realizar. La SPU y APUU fueron notificadas de esta iniciativa y se les solicitó información sobre las exigencias para la formación de los psicólogos, si éstos estarían habilitados para el ejercicio de la psicoterapia y en caso de que sí, cuáles serían las condiciones exigidas de educación y ejercicio (SPU, 1975).

La SPU respondió afirmando que el psicólogo debía ser formado como profesional con todos los privilegios y responsabilidades que esto implica, generando así independencia del orden médico. A pesar de esto, en sus propuestas siempre consideraron al menos la interconsulta y la aprobación relativa del médico para iniciar un tratamiento. Propusieron la creación de una Facultad de Psicología con estudios de grado de cinco años, especialización e investigación. Reivindicaron el ejercicio de la psicoterapia con previa capacitación, lo que requería de cursos teóricos intensivos, práctica supervisada, aprendizaje de trabajo en grupos multidisciplinarios, estudios sistemáticos y críticos de las diferentes teorías y técnicas (SPU, 1974).

La APUU respondió que el Plan 71 cumplía con los requisitos necesarios para la formación de los psicólogos, enumerando las competencias para las que se habilitaba al profesional en los ámbitos educacional, laboral, clínico y jurídico. Se destacó además el reconocimiento internacional de la profesión (UNESCO, OIT y OMS) y la necesidad de acompañar los desarrollos de otros países alentando "la creación de una Facultad de Psicología que funcionara dentro de la UdelaR como una carrera mayor, autónoma, formando profesionales independientes" (ASOCIACIÓN DE PSICÓLOGOS UNIVERSITARIOS DEL URUGUAY [APUU], 1974, p. 5). Se incluyó el ejercicio de la psicoterapia, entendida por la OIT como función del psicólogo. Por último, la APUU utilizó fragmentos del proyecto presentado al MSP por el Dr. Tobler, reforzando la necesidad de que la Licenciatura se impartiera en la FHC, y que la psicoterapia fuese parte de las labores del psicólogo, aunque implicase previa aprobación del médico tratante (APUU, 1974). Si bien ambas respuestas destacaron el ejercicio de la psicoterapia como práctica de la psicología, también incluyeron la

\footnotetext{
${ }^{10}$ La Comisión estuvo formada por el Decano Interventor de la Facultad de Medicina Dr. Gonzalo Fernández, Decano Interventor de Facultad de Humanidades y Ciencias Lic. Miguel Klappenbach y los médicos Ofelia Bachini, Carlos Tobler y Héctor Puppo integrantes de la Sociedad de Psiquiatría del Uruguay.
} 
necesidad del aval médico para iniciar el proceso, indicio de que aún no estaban dadas las condiciones para exigir exitosamente o establecer una autonomía absoluta.

El Dr. Berta también fue consultado y en julio de 1974 respondió que los estudiantes debían cumplir con los preparatorios de medicina, que la formación debía ser universitaria y no en institutos privados, de acceso restringido para no formar más psicólogos que los necesarios para "satisfacer las necesidades del país en la materia" (BERTA, 1974, p. 2). Planteó que los psicólogos son técnicos especializados que debían conocer tanto las técnicas como los límites de las mismas, diferenciándose así de los simples técnicos. Sugirió una formación teórico-práctica de cuatro años, abarcando las áreas de psicología, filosofía, biología y metodología científica. Sobre el ejercicio de la psicoterapia, sostuvo que estaría condicionada por tres elementos: formación psicológica universitaria, formación psicoterápica universitaria, $\mathrm{y}$ trabajo en equipo. Sobre este último punto, el médico-psiquiatra sería el principal responsable del funcionamiento del equipo de trabajo, ya que el psicólogo no podría "aplicar psicoterapia en forma autónoma sino por indicación y con adecuado control del médico-psiquiatra" (BERTA, 1974, p. 8).

Finalmente se resolvió el cierre definitivo de la Licenciatura en Psicología. La comisión formada para estudiar los destinos de la formación acordó en: "crear la Escuela o Instituto de Psicología, directamente dependiente de Rectoría, con un cuerpo docente seleccionado y capacitado para formar verdaderos Psicólogos" (UdelaR, 1975, p. 10), dando lugar a la creación de la Escuela Universitaria de Psicología (en adelante EUP).

En setiembre de 1975, por decreto Ministerial, se creó la EUP. El redactor del plan de formación y su primer Director el Dr. Mario Berta, expresó que su creación significó "un reconocimiento de la importancia de la Psicología como disciplina autónoma y un proceso real en su enseñanza y formación dentro de la Universidad" (BERTA, 1981, p. 2). El plan de estudios fue aprobado por el MEC en 1977 e inició los cursos al año siguiente.

La EUP no reconocía la formación específica en otras instituciones y con respecto a la formación estableció que: "las funciones del psicólogo deben quedar limitadas, en sus aplicaciones, al psicodiagnóstico. El psicólogo recibido (4 años de ciclo básico) no tiene la preparación específica para ejercer psicoterapia" (UdelaR, 1979, p. 2). Propendía a su 'rectitud' en el quehacer al formarlo para integrar equipos multidisciplinarios, para que tuviera "conciencia clara de los límites de su acción específica en relación a las otras disciplinas" y se disminuyan "los riesgos de la acción individualista, acrítica y sin control”, "mostrando las ventajas evidentes de este quehacer interdisciplinario sobre una práctica individualista, aparentemente más libre y ciertamente más peligrosa" (UdelaR, 1979, p. 4-5). El control llegaba a todos los espacios, el material de estudios se basaba en las clases grabadas e impresas de los docentes; "el material total compaginado, corregido y publicado constituirá el principal recurso bibliográfico, ya que es intención de la Escuela formar sus propias fuentes de información en cada materia" y para el ejercicio docente se estableció que: "las autoridades de la Escuela, en cumplimiento de sus funciones realizarán en cualquier momento visitas de inspección a las clases teóricas o prácticas" (UdelaR, 1979, p. 18-19).

Durante este período inicial de la dictadura, se consolidaron una serie de acciones que tendieron a delimitar el ejercicio profesional de la psicología en detrimento de los intereses de los diferentes colectivos organizados de la psicología local. Ejemplo de esto es la desaparición de una licenciatura y la creación de una escuela como ámbito de formación de un nivel académico muy inferior. La formación de la EUP aseguraba que sus egresados no tuviesen las herramientas de trabajo para ocupar las jurisdicciones en disputa, como fue el caso de la psicoterapia. La imagen de los psicólogos terminaba de refrendarse con expresiones utilizadas en el documento del Plan de estudios y reglamentaciones internas de la EUP (UdelaR, 1979), como "seudopsicólogo", "verdaderos psicólogos", desacreditando a profesionales y formaciones anteriores fuera de esta escuela, favoreciendo la visión de que ejercían tareas para las que no estaban capacitados y de las que debían ser excluidos. A esto se suma que en marzo de 1977, la Facultad de Medicina aprobó un nuevo plan de postgrado en psiquiatría dirigido a la formación en psicoterapia. Esta formación constaría de tres fases: seminario, práctica psicoterápica y aplicación psicoterápica (TUANA, 1980).

La formación de la EUP sin la capacidad de realizar psicoterapia, y la capacitación para el ejercicio de la misma comprendida en el postgrado de psiquiatría, muestran como la disputa por reglamentar el campo profesional se trasladó sobre la formación. El control ganado por la psiquiatría de este nuevo territorio reordenó los circuitos de transmisión y aplicación de la psicología, a la vez que fortaleció la autoridad del médico psiquiatra jerarquizando su posición por encima del rol del psicólogo.

\section{Declive y fin de la dictadura. Emergencia y ensamble de los colectivos de la psicología (1979-1984)}

Durante el período final de la dictadura continuó el choque entre los colectivos interesados en reglamentar el ejercicio profesional de la psicología y delimitar una jurisdicción disciplinar.

El declive del gobierno de facto fue teniendo su correlato en esa disputa. A medida que este perdía legitimidad, se fueron debilitando también los embates de sectores de la medicina que intentaban imponer su visión en la reglamentación del ejercicio de la psicología. También, al tiempo en que se avizoraba el retorno a la democracia, emergían fortalecidos diferentes colectivos de la psicología universitaria.

Un nuevo espacio de formación se crea en 1979. El 27 de marzo, el Poder Ejecutivo a través del MEC, decretó la habilitación del dictado de cursos por el Departamento de Psicología del Instituto de Filosofía, Ciencias y Letras $^{11}$ (en adelante IFICLE). Se resolvió que la Univer-

\footnotetext{
${ }^{11}$ El Instituto de Filosofia, Ciencias y Letras fue una institución de carácter privado fundada en 1954 cuyo objetivo fue la formación de profesores y profesionales en diferentes disciplinas. En 1984, a partir del último decreto de la dictadura cívico-militar se creó la Universidad Católica del Uruguay, siendo la continuidad de esta institución.
} 
sidad intervenida supervisaría la carrera y la expedición de títulos (APUU, 1979a). Este espacio se transformó en una alternativa educativa, y tuvo relativa libertad con respecto al poder dictatorial, pero también cierta independencia del poder médico.

En 1979 la APUU inició nuevas gestiones ante el MEC promoviendo la reglamentación de la profesión. En esta ocasión se argumentó que si fue el Estado quien promovió la formación en psicología, es el Estado quien debe asumir la responsabilidad en el contralor de su ejercicio profesional. El proyecto de la APUU proponía homologar con el título de Psicólogo a las titulaciones de la EUP, el IFICLE y de Técnico en Psicología Infantil de la Escuela de Tecnología Médica, y el de los egresados del Instituto de Psicología de la FHC, con el título de Licenciado en Psicología. Se proponía que los psicólogos podrían realizar tareas docentes y de diagnóstico, mientras que para los licenciados se agregaba la aplicación de "métodos y técnicas para el tratamiento posterior” (APUU, 1979b, p. 8). La APUU apostó con este movimiento a reivindicar el lugar de los egresados de la FHC dándoles potestades diferenciales frente a otros profesionales y técnicos, una acción que se sumaba a los múltiples intereses ya en juego por regular el ejercicio de la psicología, determinar potestades profesionales y jerarquizar diferentes actores dentro de una jurisdicción en pujas por ser monopolizada.

En ese mismo período, el MEC recibió un proyecto de creación de la profesión de psicólogo, enviado por el IFICLE. La propuesta legitimaba el ejercicio para todos los egresados de la UdelaR e instituciones privadas habilitadas por el MEC. Para las funciones del psicólogo destacaba el psicodiagnóstico, el asesoramiento y la docencia, nada decía sobre la psicoterapia, lo que fue cuestionado por la APUU (1979c).

El 24 de noviembre de 1979, la APUU organizó una jornada de trabajo abordando el tema del rol del psicólogo y su lugar en la tarea psicológica, contando con la presencia de 79 profesionales y 54 estudiantes. Las diferentes panelistas ${ }^{12}$ enfocaron sus presentaciones en subrayar la necesidad de regular la profesión, trabajar en la definición de los conceptos de clínica, psicólogo, psiquiatra y psicoterapeuta y criticaron las regulaciones que dan un lugar central al médico, catalogándolas como fuera de época (APUU, 1980). Las panelistas reivindicaron la formación en diferentes perspectivas psicológicas, destacando la presencia de profesionales en los más diversos ámbitos y subrayando la singularidad de su experticia integrando equipos multidisciplinarios. La identidad profesional fue tema principal de la jornada, una iniciativa estratégica dirigida a fomentar la cohesión de los colectivos de la psicología ante la proliferación de propuestas de formación y reglamentación de la profesión.

En setiembre de 1980 cumpliendo con el Decreto Ministerial de creación de la EUP, que encomendaba al Director de la misma y al Director General de Planeamiento Universitario, la elaboración de un anteproyecto

\footnotetext{
${ }^{2}$ Los panelistas fueron: Elida Tuana, Doris Hajer, Sylvia Arrambide, José Liberman, Aída Fernandez y Alba Busto.
}

de ley para la regulación del ejercicio de la psicología, los Dres. Mario Berta y Mario Grolero presentaron a consideración de la Universidad una nueva Ley del Ejercicio Profesional del Psicólogo. En una entrevista en el Diario El Día (RONZONI, 1980, p. 14) Berta expresó que la ley debe posibilitar: "que el psicólogo clínico formado como psicoterapeuta aplique técnicas de psicoterapia”, puntualizaba que esta acción debía darse en el marco de un equipo multidisciplinario integrado por un médico psiquiatra. El periodista Raúl Ronzoni (1980, p. 14) incluyó en la nota la experiencia Argentina sobre la implementación de una ley del ejercicio profesional calificada por la Federación de Psicólogos Argentina como arbitraria y limitante del ejercicio, augurando que si bien aún no se conoce el texto de la ley uruguaya: "aparentemente tendría alguna similitud con el argentino lo que seguramente crearía una dura polémica"). Desde la UdelaR se solicitó la valoración de la propuesta de la EUP a los diferentes colectivos de la psicología, el vaticinio del periodista se cumpliría en un contexto que comenzaba a dar señales de cambio.

El clima social y político en el que se enmarcó esta nueva propuesta de reglamentación, se transformaba reflejando un fuerte cuestionamiento a las diversas manifestaciones que surgieran desde los órganos del poder dictatorial. El 30 de noviembre de 1980, el gobierno cívico-militar convocó a un plebiscito con el propósito de legitimar el gobierno dictatorial a través de la sustitución de la Constitución de 1967. La población rechazó la propuesta con cerca de un $57 \%$, lo que a la postre abrió el camino a un proceso de reapertura democrática que se continuó en 1982 con elecciones de los partidos políticos habilitados ${ }^{13}$, negociaciones entre los partidos políticos y los militares durante 1983 y 1984, para finalizar con las elecciones democráticas el 25 de noviembre de 1984.

Las respuestas a la propuesta de reglamentación elaborada por Berta y Grolero comenzaron a llegar marcando un tono firme y contrario a la misma. La Escuela de Tecnología Médica respondió la consulta a través de su Director, el Dr. Sica y el Dr. Alterwain encargado del curso de Psicología Infantil; planteando que se desconoce la formación en psicología de distintos espacios universitarios y solo reconoce a la EUP, cuya formación no capacita específicamente para el área de la salud. Se propone la inclusión de los técnicos en Psicología Infantil como psicólogos universitarios y su inscripción en el MSP junto a los especialistas en Psicología Clínica, a diferencia de los egresados de la EUP que deben inscribirse para su ejercicio en el MEC y no se comparte la exigencia por ley de la integración a equipos multidisciplinarios (ASOCIACIÓN DE PSICÓLOGOS UNIVERSITARIOS EGRESADOS DE LA ESCUELA DE TECNOLOGÍA MÉDICA [APUEETM], 1983).

En esa misma institución, el 13 de noviembre de 1981, se fundó la Asociación de Psicólogos Universitarios Egresados de la Escuela de Tecnología Médica (en adelante APUEETM). La creación se dio en el marco de una situación que los asociados calificaron como ame-

${ }^{13}$ Los partidos políticos habilitados fueron el Partido Colorado, el Partido Nacional y la Unión Cívica, mientras que no fue habilitado el Frente Amplio. 
nazante para la profesión. La APUEETM (1983, p. 15) respondió al rector interventor Dr. Hector Viana Reyes haciendo énfasis en que el anteproyecto se dirige a: "evitar interferencias técnicas y sociales con otras profesiones", también subrayó su carácter limitante y abordaron con claridad lo que denominaron: 'tratamiento psicológico', para el que se utilizan técnicas propias de la psicología, prueba de ello es que "los médicos psiquiatras que en nuestro medio se dedican al tratamiento psicológico, han debido formarse para ello de manera independiente a su carrera médica" (APUEETM, 1983, p. 17). En razón de esto entienden como totalmente irrelevante que el psicólogo deba estar bajo dirección de un médico psiquiatra para realizar un tratamiento psicológico como se plantea en el Art 4 del anteproyecto.

El 15 de octubre de 1981, la SPU contestó a la solicitud realizada el 15 de setiembre (SPU, 1982a), reafirmando la identidad profesional del psicólogo y la necesidad de crear una Facultad de Psicología en la UdelaR. Se cuestionó el error de considerar sólo a la EUP como espacio de formación universitaria y reafirmó las competencias de los psicólogos para dirigir servicios de psicología, realizar pericias y asesoramientos. En relación a la psicoterapia, sostuvo que el psicólogo podrá realizarla según se determine en la reglamentación y en consulta entre psicólogo y médico. Se cuestionó que el MEC por sí solo tenga la potestad de habilitar establecimientos universitarios privados, dejando de lado al ente competente que sería la UdelaR. El anteproyecto de la EUP delegaba sobre autoridades políticas responsabilidades inherentes a espacios de enseñanza superior. Se señaló duramente el modo de entender al psicodiagnóstico, solo como aplicación de tests, simplificación que desjerarquiza la labor técnica e intenta fijar una posición de subordinación en relación al médico, quien sería el referente de la intervención tanto para el diagnóstico como para el trabajo del psicólogo clínico. La Directiva de la SPU consideró inadmisible esa postura y sentenció: "no pueden aceptarse profesionales de segundo orden, cuya conducción profesional y responsabilidad laboral deban ser asumidos por otro, de otra disciplina que se erija en censor" (SPU, 1982a, p. 10).

El 18 de noviembre de 1981, luego de recibir las diferentes opiniones de los colectivos de psicólogos, el Rector Interventor comunicó la resolución que adoptó subrayando que la mayoría de de las respuestas fueron de rechazo, dando muestra de objeciones válidas de la forma y contenido del anteproyecto. Se consideró que este último debía desecharse y disponerse la elaboración de uno nuevo que respondiese al interés general. Se resolvió conformar un grupo integrado por Directores Generales de Servicios Jurídicos y de Planeamiento Universitario y el Secretario Docente de la EUP que para su labor, recabara las opiniones de los diferentes colectivos vinculados a la psicología (SPU, 1982a).

El Estudio del Anteproyecto en la UdelaR no se continuó, el cambio del Rector de la Universidad y la renuncia del Dr. Mario Berta por causal jubilatorio fueron determinantes en la no continuidad de esa tarea. De todas mane- ras, la SPU (1982b) no perdió la oportunidad de solicitar que la Dirección de la EUP fuera asumida por un psicólogo. A pesar que el tema instalado refería a la legislación del ejercicio profesional, en diciembre de 1983, la EUP invitó a los colectivos ligados a la psicología a conversar sobre el problema de la formación profesional de los psicólogos. Los primeros años de la década de 1980 reflejaban el declive de los impulsos autoritarios y emergía un escenario distinto al de los inicios de la dictadura en 1973.

En el segundo semestre de 1983 se crearon la Asociación de Estudiantes y Egresados de la Escuela Universitaria de Psicología y la Asociación de Psicólogos Egresados del IFICLE. El 28 de octubre de 1983 se reunieron por primera vez todas las asociaciones de egresados de psicología del país, de ahí en más las acciones que siguieron al final de la dictadura e inicios del período democrático tuvieron el objetivo de aunar fuerzas y forjar un destino común en la formación universitaria y en la regulación del ejercicio profesional (SPU, 1983). Este impulso logró la creación de la Coordinadora de Psicólogos en octubre de 1987, asociación profesional de todos los psicólogos universitarios que llevó adelante junto con la Facultad de Psicología el proceso de reglamentación de la profesión que culminó con la sanción de la ley N. ${ }^{\circ} 17.154$ (URUGUAY, 1999). El período democrático iniciado en 1984 queda fuera de nuestra investigación actual, por lo que se continuará en estudios próximos el proceso que llevó a alcanzar el logro tan ansiado para la psicología universitaria de legislar sobre el ejercicio profesional.

\section{Conclusiones}

Al inicio planteamos que las controversias en el ámbito de las disciplinas psi en Uruguay, se dan cuando se intenta regular un espacio fronterizo mayormente entre sectores de la psicología y la psiquiatría. Abbott (1988) denomina jurisdicción a la conexión que vincula a una profesión con el trabajo que realiza, en este artículo describimos y analizamos el modo en que una jurisdicción es reclamada en el terreno formal (el espacio público y legal), visualizando a partir de esa aproximación que la disputa por una jurisdicción no se reduce a un enfrentamiento entre profesiones, sino que pone en juego un vasto entramado relacional de mucha complejidad. El entorno de cada una de esas disciplinas y sus articulaciones con otros sectores (gobierno, mercado, agrupaciones), forman las amplias redes que los sujetos colectivos elaboran con sus acciones y argumentos (DANZIGER, 1984), a la vez que consolidan posiciones en las controversias y materializan procesos de profesionalización de la psicología (ROSE, 1998).

El entorno que emerge a partir de los movimientos de los actores implicados, resulta un territorio donde diferentes dominios parecen pasibles de ser conquistados, pueden ser espacios de formación profesional, instancias de habilitación al ejercicio de las profesiones, organismos de gobierno, entre otros. En este sentido, si bien el foco de las controversias se situó en el intento de reglamentar el ejercicio profesional de la psicología y el núcleo fue el ejercicio específico de la psicoterapia, el interés de los 
diferentes colectivos por codificar un espacio que involucra a todo el entorno se vuelve estratégico. Esto nos lleva a pensar que la disputa por el uso de ciertas técnicas, por los perfiles de la identidad profesional y los modos de circulación, apropiación y aplicación del conocimiento, expresa a la vez el enfrentamiento por campos más amplios, como el de la salud mental para el caso de las disciplinas "psi". En razón de esto, se vuelve importante reconocer una procedencia genealógica de los actuales campos de disputa, historia y actualidad se vuelven aspectos necesarios para comprender la construcción de las profesiones, sus relaciones y la legitimidad de sus jurisdicciones.

\section{Informações sobre os autores:}

\section{Jorge Chavez}

\section{iD https://orcid.org/0000-0002-8123-6431}

Doctor en Psicología por la Universidad Nacional de Córdoba (Argentina). Es Licenciado en Psicología. Licenciado en Ciencias de la Educación y Magíster en Psicología y Educación por la Universidad de la República. Es Profesor Agregado de la Facultad de Psicología de la Universidad de la República, docente en Régimen de Dedicación Total e integrante del Sistema Nacional de Investigadores (SNI). Es responsable del Grupo de Investigación: Estudios de la Gubernamentalidad, Ciencia, Tecnología y Subjetividad (EGCTS) de la Universidad de la República.x

\section{Paula Martínez Sena}

iD https://orcid.org/0000-0002-2983-3327

Licenciada en Psicología por la Universidad de la República. Acompañante Terapéutica por Universidad CAECE (Argentina). Actualmente cursando formación en Sexología en la Sociedad de Estudios Superiores de Sexología (SESSEX) y Curso de Posgrado "Asistencia integral a varones que ejercen violencia de género" por la Universidad Provincial de Córdoba (Argentina).

\section{Contribuições dos autores:}

Todos os autores colaboraram ao longo do processo, desde a elaboração até a revisão final do manuscrito. Ambos aprovaram o manuscrito final para publicação.

\section{Como citar este artigo:}

\section{ABNT}

CHAVEZ, Jorge; MARTINEZ SENA, Paula. Controversias sobre el ejercicio profesional de la psicología en Uruguay. Fractal: Revista de Psicologia, Niterói, v. 33, n. 2, p. 151-161, maio/ago. 2021. https://doi.org/10.22409/1984-0292/v33i2/49362

\section{APA}

Chavez, J., \& Martinez Sena, P. (2021, Maio/Agosto). Controversias sobre el ejercicio profesional de la psicología en Uruguay. Fractal: Revista de Psicologia, 33(2), 151-161. doi: https://doi. org/10.22409/1984-0292/v33i2/49362

\section{Copyright:}

Copyright (C) 2021 Chavez, J., \& Martinez Sena, P. Este é um artigo em acesso aberto distribuído nos termos da Licença Creative Commons Atribuição que permite o uso irrestrito, a distribuição e reprodução em qualquer meio desde que o artigo original seja devidamente citado.

Copyright (C) 2021 Chavez, J., \& Martinez Sena, P. This is an Open Access article distributed under the terms of the Creative Commons Attribution License, which permits unrestricted use, distribution, and reproduction in any medium, provided the original article is properly cited.

\section{Referencias}

ABBOTT, Andrew. The system of professions: an essay on the division of expert labor. Chicago: The University of Chicago Press, 1988.

ASOCIACIÓN DE PSICÓLOGOS UNIVERSITARIOS DEL URUGUAY. Boletín Informativo, Año 3, n. 3, Montevideo, 1973.

ASOCIACIÓN DE PSICÓLOGOS UNIVERSITARIOS DEL URUGUAY. Respuesta de la APUU al pedido del Señor Decano Interventor de la Facultad de Medicina sobre aspectos fundamentales acerca de la formación y el ejercicio profesional del Psicólogo (mimeografiado). Archivo Mario Berta. Montevideo: Facultad de Medicina, UdelaR, 1974.

ASOCIACIÓN DE PSICÓLOGOS UNIVERSITARIOS DEL URUGUAY. Nueva carrera de Psicología reconocida oficialmente. Revista Uruguaya de Psicología, Montevideo, v. 1, n. 2, p. 78, 1979a.

ASOCIACIÓN DE PSICÓLOGOS UNIVERSITARIOS DEL URUGUAY. Gestión de APU ante el Ministerio de Educación y Cultura. Revista Uruguaya de Psicología, Montevideo, v. 1, n. 3, p. 7-9, 1979b.

ASOCIACIÓN DE PSICÓLOGOS UNIVERSITARIOS DEL URUGUAY. Proyecto de creación de la profesión de psicólogo. Revista Uruguaya de Psicología, Montevideo, v. 1, n. 3, p. 107108, 1979c.

ASOCIACIÓN DE PSICÓLOGOS UNIVERSITARIOS DEL URUGUAY. Rol del Psicólogo. Revista Uruguaya de Psicología, Montevideo, v. 1, n. 4, p. 11-41, 1980.

ASOCIACIÓN DE PSICÓLOGOS UNIVERSITARIOS EGRESADOS DE LA ESCUELA DE TECNOLOGÍA MÉDICA. Fort- da: Publicación de la Asociación de Psicólogos Universitarios Egresados de la Escuela de Tecnología Médica. Montevideo: [s.n.], 1983.

BECHER, Tony. Tribus y territorios académicos. Barcelona: Gedisa, 2001.

BERTA, Mario. Límites y campo de acción de la Psicología en relación con la Psiquiatría. Montevideo: Talleres Gráficos Goes, 1958.

BERTA, Mario. Respuesta al Señor Decano Interventor Prof. Dr. Gonzalo Fernández, Facultad de Medicina. Archivo Mario Berta. Montevideo: Facultad de Medicina, UdelaR, 1974.

BERTA, Mario. Informe sobre creación y evolución de la EUP solicitado por la Oficina de Planeameinto y Presupuesto de la UDELAR. Archivo Mario Berta. Montevideo: Facultad de Medicina, UdelaR, 1981.

CARRASCO, Juan Carlos. Palabras de Clausura. Primeras Jornadas Nacionales de Psicología Infantil. Montevideo: Comunidad del Sur, 1967. p-179-181.

CASSINELLI, Horacio. Informe. Archivo Mario Berta. Montevideo: Facultad de Medicina, UdelaR, 1972.

CHAVEZ, Jorge; IRRAZABAL, Enrico. La psicología social universitaria en Uruguay: Aportes para una historia crítica. In: JACÓ-VILELA, Ana María (Org.). Psicología Social: itinerários na América Latina. Curitiba: Juruá, 2018. p. 223-246. 
CHAVEZ, Jorge. Genealogía de las prácticas en el campo de la psicología y su relación con las tecnologías de gubernamentalidad en el Uruguay moderno: un enfoque desde los estudios en ciencia, tecnología y sociedad. 2020. $495 \mathrm{f}$ Tese (Doutorado em Psicologia) - Universidad Nacional de Córdoba, Córdoba, 2020.

DANZIGER, Kurt. Hacia un marco conceptual para una historización crítica de la psicología. Revista de Historia de la Psicología, v. 5, n. 1-2, p. 99-107, 1984. Disponível em: https://www.studocu.com/cl/document/universidad-diegoportales/historia-de-la-psicologia/resumenes/hacia-un-marcoconceptual-para-una-historizacion-critica-de-la-psicologialisto/4096191/view. Acceso em: 19 feb. 2018.

FACULTAD DE HUMANIDADES Y CIENCIAS. Informe del Dr. Semino sobre el proyecto de reglamentación de la carrera de psicología. Archivo Mario Berta. Montevideo: Facultad de Medicina, UdelaR, 1973a

FACULTAD DE HUMANIDADES Y CIENCIAS. Expediente $n^{\circ}$ 381927. 10 de setiembre de 1973. Archivo Mario Berta Montevideo: Facultad de Medicina, UdelaR, 1973b.

FACULTAD DE MEDICINA. Expediente $n^{\circ} 752123$. Asunto: Ministerio de Salud Pública. Depto. de psiquiatría e higiene ambiental establece normas para las actividades específicas de psicólogos y psicotécnicos en todo el territorio nacional. 23 de abril de 1975. Archivo Mario Berta. Montevideo: Facultad de Medicina, UdelaR, 1975.

FREIDSON, Eliot. Para uma análise comparada das profissões a institucionalizaçao do discurso e do conhecimiento formais. Revista Brasilera de Ciências Sociais, São Paulo, v. 11, n. 31, p. 141-154, 1996. Disponível em: https://cff.org.br userfiles/60\%20-\%20FREIDSON\%20E\%20\%20Parna $\% 20$ uma $\% 20$ aalise $\% 20$ comparada\%20das\%20profissoes 1996 . pdf. Acceso em: 19 feb. 2018

JIMENEZ DE ARECHAGA, Justino. Informe. Archivo Mario Berta. Montevideo: Facultad de Medicina, UdelaR, 1972.

LIJTENSTEIN, Marcos; VIÑAR, Marcelo. Entrevista realizada a Willy y Madelaine Baranger. Revista Uruguaya de Psicoanálisis, Montevideo, v. 76, p. 273279, 1992. Disponível em: http://www.apuruguay.org/ apurevista/1990/1688724719927629.pdf. Acceso em: 20 mar. 2018.

MARCOS, Julio. La clínica Médico-Psicológica del Hospital de Niños "Dr. Pedro Visca". Montevideo: Rosgal, 1949.

OIBERMAN, Alicia. Pioneros de la psicología en Argentina: Historias de vida...cuando la vida es historia. Entrevista realizada a la Prof. Elida Tuana y al Prof. Juan Carlos Carrasco, 2001. Disponível em: http://www.palermo.edu/cienciassociales/ psicologia/publicaciones/pdf/Psico3/3Psico\%2003.pdf. Acceso em: 9 out. 2019.

REYES TERRA, José María. El problema del Intrusismo en Psiquiatría en el Uruguay. In: Psicología: Universidad e Historia de la Psicología. Montevideo: Multiplicidades, 1958a. p. 37-50.

REYES TERRA, José María. Palabras pronunciadas por el Dr. Jose M. Reyes Terra en la audiencia concedida por el Sr. Ministro de Salud Pública a un grupo de miembros de la Sociedad de Psiquiatría del Uruguay el 12/6/1958 (mimeografiado). Archivo Mario Berta. Montevideo: Facultad de Medicina, UdelaR, 1958b.
RICO, Alvaro. 15 dias que estremecieron al Uruguay: golpe de Estado y huelga general: 27 de julio-11 de julio de 1973. Montevideo: Fin de Siglo, 2006.

RICO, Alvaro et al. La caída de la democracia: cronología comparada de la historia reciente del Uruguay (1967-1973). Montevideo: Fundación de Cultura Universitaria, 1996.

RONZONI, Raúl. Estatuto Jurídico para el Ejercicio de la Psicología. El Día, Montevideo, p. 14-15, 5 oct. 1980.

ROSE, Nikolas. A critical history of psychology. In: Inventing our selves: psychology, power, and personhood. Cambridge: Cambridge University Press, 1998. p. 41- 66.

SOCIEDAD DE PSICOLOGÍA DEL URUGUAY. Citación Asamblea General Extraordinaria (mimeografiado). Archivo Mario Berta. Montevideo: Facultad de Medicina, UdelaR, 1973.

SOCIEDAD DE PSICOLOGÍA DEL URUGUAY. Hoja informativa n. 30-31, Montevideo, 1974.

SOCIEDAD DE PSICOLOGÍA DEL URUGUAY. Hoja informativa n. 34-35, Montevideo, 1975.

SOCIEDAD DE PSICOLOGÍA DEL URUGUAY. Boletín de la Sociedad de Psicología del Uruguay, Montevideo, p. 54-55, 1982a.

SOCIEDAD DE PSICOLOGÍA DEL URUGUAY. Boletin de la Sociedad de Psicología del Uruguay, Montevideo, p. 56-57, enero/jun. 1982b.

SOCIEDAD DE PSICOLOGÍA DEL URUGUAY. Boletín de la Sociedad de Psicología del Uruguay, Montevideo, p. 62-63, agosto/dic. 1983.

TUANA, Elida. Reconocimiento legal del campo profesional del psicólogo. Revista Uruguaya de Psicología, Montevideo, v. 1, n. 4, p. 12-18, 1980.

UNIVERSIDAD DE LA REPÚBLICA. Expediente 3651/75. 6 de junio de 1975. Consejo de Estado eleva nota a Rector de la Universidad referente memorándum relacionado con la cátedra de psicología de la Facultad de Humanidades y Ciencias. Montevideo: Udelar, 1975

UNIVERSIDAD DE LA REPÚBLICA. Plan de estudios 1977 y reglamentaciones internas 1979. Montevideo: Dirección General de Extensión Universitaria. Depto de Publicaciones y Ediciones, 1979.

URUGUAY. Poder Legislativo. Ley $N^{\circ}$ 17.154. Dictanse normas relativas al ejercicio de la profesión de psicólogo. 27 agosto 1999. Disponible en: https://legislativo.parlamento.gub.uy/ temporales/leytemp4412998.htm. Acceso en: 12 marzo 2021. 